patients infected worldwide. The, UKmost common genotype of HCV is Genotype 1. For many years, the 'Standard of Care' has been prolonged therapy with the combination of Polyethylene glycol interferon- $\alpha$ and Ribavirin for one year. The recent approval of drugs such as Telaprevir and Boceprevir has restructured treatment for genotype $1 \mathrm{HCV}$ infection, both for previously treated or treatment-naïve patients. Specific dietary intake is warranted for adequate absorption of the protease inhibitors and this has led to a structured dietary advice being given to patients. We wanted to compare the nutritional impact of antiviral therapy in two groups of patients who underwent either dual or triple therapy.

Methods Treatment of hepatitis C patients at Aberdeen Royal Infirmary is undertaken by Hepatology specialist nurses who review these patients at designated time intervals. At each of these clinic visits the MUST score (Malnutrition Universal Screening Tool) is recorded in addition to the weight and BMI of the patient. Any patient who has a MUST score of 2 is referred for specialist dietetic input, which is also available at the clinic. At 4 weekly intervals, the HAD score for anxiety and depression is also recorded for all patients.

Results A total of 73 patients with Genotype 1 underwent therapy in the year 2012-2013, including 25 patients with dual therapy of PEG-IFN and Ribavirin and 48 patients on triple therapy of PEG-IFN, Ribavirin and Telaprevir. There was no statistical difference in the initial weight, BMI and MUST scores of these two groups of patients. The mean weight fell from $89.6 \pm 17.9$ $\mathrm{kg}$ to $83.7 \pm 15.6 \mathrm{~kg}$ by the end of treatment in the dual therapy group with a parallel fall in BMI from $30.7 \pm 5.4$ to 28.8 $\pm 4.6 \mathrm{~kg} / \mathrm{m}^{2}$. However, in the triple therapy group, the mean weight increased from $82.2 \pm 30.5 \mathrm{~kg}$ to $82.7 \pm 26.0 \mathrm{~kg}$ and a stable BMI from $26.4 \pm 9.5$ to $26.2 \pm 8.3 \mathrm{~kg} / \mathrm{m}^{2}$. Dietary referral and intervention was needed in $7 / 25$ patients on dual therapy $(28 \%)$ as opposed to $7 / 48$ patients on triple therapy (14.6\%) (p $=0.23)$

Conclusion Weight reduction is a significant problem in patients undergoing dual therapy for hepatitis $\mathrm{C}$ as opposed to those undergoing triple therapy. Referral to the dietician was needed in a larger proportion of cases undergoing standard of care though it did not reach statistical significance. Dietary advice given with respect to protease inhibitors may have had a significant effect in combating the ill effects of the standard treatment of patients with Genotype $1 \mathrm{HCV}$ infection. This specific advice should be extrapolated to all patients undergoing anti-viral treatment.

Disclosure of Interest None Declared.

\section{PTH-085 SYSTEMATIC REVIEW OF PERFORMANCE OF ALT IN PREDICTING LIVER FIBROSIS: ALT IS A SENSITIVE, BUT NOT A SPECIFIC MARKER OF LIVER FIBROSIS IN POPULATIONS WITH/SUSPECTED LIVER DISEASE}

'f Coad*, 'S Olsen, 'W Rosenberg, 'P Roderick, 'I Parkes. 'University Hospital Southampton, Southampton, UK; ${ }^{2}$ University College London, London, UK

\subsection{6/gutinl-2014-307263.531}

Introduction ALT is one of the most commonly used tests to detect liver disease for further investigation, but its accuracy is uncertain. Currently there is no systematic review of diagnostic accuracy of ALT in detecting liver fibrosis using liver biopsy as reference standard.

Methods Standardised methods for conducting systematic reviews were used. MEDLINE, EMBASE, and reference lists from articles were searched. Studies were included if they

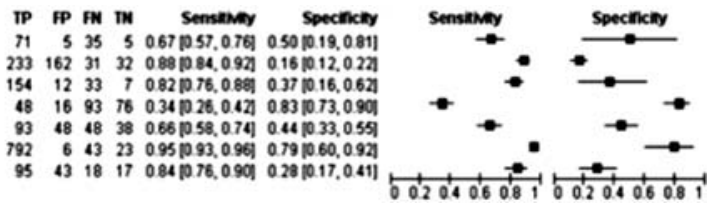

Abstract PTH-085 Figure 1

evaluated paired samples of liver biopsy and serum, with extractable data for ALT, $>30$ participants, and presented data as sensitivity, specificity, predictive values, likelihood ratios, or ROC curves or included sufficient data to calculate these parameters. ALT above upper limit of normal threshold were defined using local values and ranged from 30 to $60 \mathrm{u} / \mathrm{l}$.

Results Initial search located 9563 abstracts, 344 papers were assessed, of which 9 met inclusion criteria. The majority of included studies were published in last 10 years, were from secondary specialist care, and conducted in USA (3), Europe (3), and rest of world (3). Median study size was 206 (IQR 124341). Median proportion of moderate/severe fibrosis on biopsy was $22 \%$ (range 6-34), any fibrosis $81 \%$ (range 58-100). Pooled sensitivity for ANY fibrosis was $87 \%$ (95\% CI: $85-88)$ specificity 34\% (95\% CI: 30-38), positive likelihood ratio 1.3 (95\% CI: 1.2-1.4). Moderate/severe fibrosis sensitivity was $89 \%$ (95\% CI: 87-91), specificity 35\% (95\% CI: 32-37), positive likelihood ratio 1.36 (95\% CI: 1.3-1.42). AUROC values were reported in 2 studies - moderate/severe fibrosis AUROC $=0.815$, and severe fibrosis AUROC $=0.59$.

Conclusion ALT accuracy in predicting fibrosis has only been studied in specialist clinic populations where it has low specificity for both any or moderate/severe fibrosis, but high sensitivity for both. Predictive values in clinic populations with prevalences found in these studies show that ALT has high PPV for any fibrosis (because of high prevalence) but low NPV. In contrast it has high NPV for ruling out moderate/severe fibrosis but poor PPV. Results from this review suggest that ALT should be used in conjunction with other tests and clinical features and not alone to identify liver fibrosis in clinic populations. Spectrum effects are likely to be important especially in those with no fibrosis so affecting the specificity. Further studies are needed in primary care populations of the accuracy of ALT versus robust non invasive markers, but these findings would suggest that a normal ALT can rule out moderate/severe fibrosis with reasonable certainty due to its high sensitivity and likely low prevalence in primary care though this may not be the case for mild fibrosis.

Disclosure of Interest None Declared.

\section{PTH-086 COMPARISON OF CLINICAL PRESENTATIONS AND OUTCOME OF HEPATOCELLULAR CARCINOMA BETWEEN HEPATITIS C AND NONALCOHOLIC FATTY LIVER CIRRHOSIS: A SINGLE CENTRE EXPERIENCE}

${ }^{1} \mathrm{NN}$ Than*, ${ }^{2} \mathrm{~N}$ Tehami, ${ }^{3}$ J Hodson, 'S Shetty. ${ }^{1}$ Centre for Liver Research and NIHR BRU, University of Birmingham and University Hospital Birmingham NHS Trust, UK; ${ }^{2}$ Liver, Birmingham, UK; ${ }^{3}$ University Hospital Birmingham NHS Trust, Birmingham, UK

\subsection{6/gutjnl-2014-307263.532}

Introduction Hepatocellular carcinoma (HCC) is the most common primary liver tumour, and represents the third leading cause of cancer death worldwide. ${ }^{1,2}$ The most important risk factor is liver cirrhosis, mainly due to chronic infections such as hepatitis $\mathrm{B}$ or $\mathrm{C}^{2}$ Increasing HCC cases are seen in nonalcoholic fatty liver disease (NAFLD). 
Methods The aim of the study was to compare demographics, treatments and survival among hepatitis $\mathrm{C}$ virus (HCV/HCC) and NAFLD (NAFLD/HCC) cohort of patients. Data were collected from medical electronic case notes, imaging reports and HCC multidisciplinary meetings.

Results Among 292 patients, 212 patients (73\%) had underlying $\mathrm{HCV} / \mathrm{HCC}$ and 80 patients $(27 \%)$ had NAFLD/HCC. The median age at diagnosis was significantly higher in NAFLD/HCC $(\mathrm{p}<0.001)$. The majority $(82 \%)$ were male. Body mass index (BMI) was significantly higher in NAFLD/HCC $(\mathrm{p}<0.001)$. The majority were Caucasian (96\%) in NAFLD/HCC, whilst the $\mathrm{HCV} / \mathrm{HCC}$ cohort was significantly more ethnically diverse $(\mathrm{p}<$ 0.001). Diabetes mellitus was more common in NAFLD/HCC patients $(\mathrm{p}<0.001)$.

The median alpha fetoprotein level in HCV/HCC patients were 32.0 compared to 12.0 in $\mathrm{NAFLD} / \mathrm{HCC}(\mathrm{p}=0.085)$. Patients with HCV/HCC were significantly more likely to be transplanted during the study period than NAFLD/HCC $(30 \%$ vs. $15 \%, \mathrm{p}=0.010)$. Both transarterial chemoembolization (TACE) and percutaneous ethanol injection (PEI) were significantly more likely to be used as a single treatment in NAFLD patients, compared to HCV patients $(\mathrm{p}=0.042, \mathrm{p}=0.021)$. Sorafenib was used as the only treatment in $6 \%$ of HCV/HCC and $3 \%$ of NAFLD/HCC cohorts $(\mathrm{p}=0.364)$. Post transplant survival appeared to be worse in HCV-HCC patients compared to NAFLD/HCC, although it did not reach statistical significance $(\mathrm{p}=0.081)$. Overall five year survival rates were similar between the two groups regardless of any treatment therapies ( $p$ $=0.424$ ).

Conclusion Despite the NAFLD/HCC being older and with higher metabolic risk factors, a significant proportion could undergo active therapy. Furthermore, patients with NAFLD/ HCC selected for transplantation seemed to have better long term outcomes, possibly due to stricter selection for transplantation as well as variations in tumour biology between the two groups.

\section{REFERENCES}

1 Mittal S, El-Serag HB. Epidemiology of hepatocellular carcinoma: consider the population. J Clin Gastroenterol 2013;47 Suppl:S2-6

2 Lu T, et al. Prevention of hepatocellular carcinoma in chronic viral hepatitis B and C infection. World J Gastroenterol 2013;19(47):8887-8894

Disclosure of Interest None Declared.

\section{PTH-087 PERCEPTIONS OF LIVER DISEASE AMONGST THE NEPALI COMMUNITY; DESIGNING EFFECTIVE CASE-FINDING STRATEGIES TO TEST UK MIGRANT GROUPS FOR HBV AND HCV}

${ }^{1} \mathrm{M}$ Petrova, ${ }^{2} \mathrm{~J} \mathrm{Hendy,}{ }^{3} \mathrm{~J} Z$ Zamani, ${ }^{3} \mathrm{M}$ Dunstall, 'S Mathew*, ${ }^{4} \mathrm{~N}$ Margot, ${ }^{1} \mathrm{P}$ Berry, ${ }^{5} \mathrm{G}$ Foster, ${ }^{3} \mathrm{M}$ Lisa, ${ }^{6} \mathrm{P}$ Kennedy, ${ }^{7} \mathrm{~A}$ Ala. ${ }^{1}$ Gastroenterology and Hepatology, Frimley Park Hospital, Frimley, UK; ${ }^{2}$ Health Care Management and Policy, University of Surrey, Surrey, UK; ${ }^{3}$ Research and Development, Frimley Park Hospital, Frimley, UK; ${ }^{4}$ Public Health UK, Public Health Surrey, Surrey, UK; ${ }^{5}$ Gastroenterology and Hepatology, Royal London Hospital, London, UK; ${ }^{6}$ Centre for Digestive Diseases, Royal London Hospital, London, UK; ${ }^{7}$ Frimley Park Hospital, Frimley, UK

\subsection{6/gutjnl-2014-307263.533}

Introduction Identifying at-risk migrant groups for Hepatitis $\mathrm{B}$ and C (HBV and HCV) is well established. The UK Nepali community has grown rapidly since 2004, when settlement rights were given for ex-Gurkha servicemen and dependants. Given military associations, the Hampshire County now has the second largest Nepali population, with the Nepali now making up close to $10 \%$ of the population. Nepal sits between China and India, two countries with higher prevalence rates of HBV and HCV, but relatively little is known about prevalence in the Nepali community, with no published studies in the UK.

Methods To help design a culturally sensitive testing strategy for $\mathrm{HBV}$ and HCV (as advocated by NICE) we conducted focus groups sessions in the Nepali community. Nepali moderators guided sessions to study the beliefs, understanding and perceptions towards liver disease.

Results 32 Nepali members attended the focus group sessions, with groups divided by sex and age (<30yrs, or $>30 \mathrm{yrs})$. A thematic analysis approach was used to analyse results.

Perceptions of Liver disease:

"It is not a communicable disease"

"In Nepal water is the main cause of hepatitis"

"Mainly alcoholic and smokers get this disease"

"I do not think people hate the person....it would not be considered as bad as leprosy disease"

Treatment options:

"In Nepal herbal medicine is better for jaundice....necessary to drink lot of water and fruits"

Knowledge and outreach:

"We need to know the function of liver. Then we understand the issue."

"Newspaper for the people who can read but for us who cannot read, radio and TV is better"

"What the doctor said, we trust on it"

Conclusion NICE guidelines advocate testing at-risk migrant groups for HBV and HCV at an early (asymptomatic) stage. Here, all groups identified liver disease with jaundice or symptoms. Different viewpoints were expressed based on age; younger Nepali members associating a greater stigma to liver disease and hepatitis. All groups expressed a sincere wish to gain greater knowledge about liver disease and to interact with primary care. The study also identified the functional illteracy of many Nepali, and the potential need to modify approaches away from written media.

\section{REFERENCE}

1 NICE (National Institute for Health and Care Excellence), Hepatitis B and C, ways to promote and offer testing to people at increased risk of infection, 2012

Disclosure of Interest None Declared.

\section{PTH-088 NON INVASIVE MONITORING OF FATTY LIVERS IN MORBIDLY OBESE PATIENTS: PRELIMINARY EVALUATION WITH ACOUSTIC RADIATION FORCE IMPULSE IMAGING}

${ }^{1} \mathrm{~S}$ Chakravartty*, ${ }^{2} \mathrm{O}$ Jaffer, ${ }^{3} \mathrm{Y}$ Zen, ${ }^{2} \mathrm{~J}$ Dent, ${ }^{2} \mathrm{~J}$ Clarke, ${ }^{2} \mathrm{P}$ Sidhu, ${ }^{1} \mathrm{~A}$ Patel. ${ }^{1}$ Surgery, King's College Hospital, London, UK; ${ }^{2}$ Radiology, King's College Hospital, London, UK; ${ }^{3}$ Histopathology, King's College Hospital, London, UK

\subsection{6/gutjnl-2014-307263.534}

Introduction Liver biopsy remains the gold standard for diagnosing non-alcoholic steatohepatitis (NASH). But with a variability of $10-20 \%$ and a mortality rate of $0.01 \%$, non invasive techniques of monitoring change in liver morphology have been sought after. Acoustic radiation force impulse imaging (ARFI) is a promising innovation that combines ultrasound imaging and elastography to measure liver stiffness which correlates well with liver fibrosis. Morbidly obese patients are at a risk of developing NASH or Non Alcoholic Fatty Liver Disease (NAFLD) and weight loss helps improve liver steatosis. Very low calorie diets 